\title{
CHAPTER SIX \\ ARCHAEOLOGICAL INFERENCE AND THE EXPLANATION OF HOMINID EVOLUTION
}

\author{
MARK LAKE
}

Archaeology has a vital role to play in the wider palaeoanthropological endeavour to understand hominid evolution since it provides the only direct evidence for the behaviour of our ancestors and their relatives. As Glynn Isaac (1972, 1976a, 1983) made clear, the ultimate goal of hominid archaeology is to stimulate and test explanations for hominid evolution. Over the past twenty years researchers have sought to accomplish this ultimate goal through the intermediate goal of behavioural reconstruction (e.g. Isaac 1978). While very real advances have been made in identifying specific hominid activities, it is still proving difficult to reconstruct the wider behavioural context of such activities. The source of this difficulty has been identified by recent work on the structure of the East African Lower Palaeolithic archaeological record which suggests that the goal of behavioural reconstruction is not commensurable with the nature of the available evidence. This chapter assesses the implications of the mismatch between goals and data for the attempt to explain hominid evolution. First, a review of the current inferential strategy leads to the conclusion that inference about the wider organization of hominid activities is unreliable owing to a debilitating lack of temporal resolution. The second section argues that progress will require a greater understanding of the mechanisms and rates of hominid behavioural change. Only then will it be possible to establish whether the conflation of behavioural episodes across tens of thousands of years is acceptable. The final section argues that computer simulation has a vital role to play in exploring the mechanisms underlying behavioural change, and thus in assessing the viability of behavioural reconstruction as a route to the explanation of hominid evolution.

\section{INFERRING HOMINID BEHAVIOUR}

The origin of the current goals and inferential framework of early hominid archaeology can be traced back to the early 1970s, when Glynn Isaac joined Richard Leakey's exploration of fossil hominid bearing strata in the east Turkana Basin in northern Kenya. There have undoubtedly been important developments over the past two decades, but these essentially represent refinements in method rather than a radical alteration of Isaac's project. Isaac aimed to reconstruct the 'basic features of Early Pleistocene life' (1969:8). It is in this sense that current goals were referred to as 'reconstructionist', which is not to deny that Isaac was interested in wider evolutionary questions - indeed, Isaac's recognition of the need to test models of human evolution against the 
archaeological record will surely prove to be one of his most enduring contributions to palaeoanthropology Rather, the term 'reconstructionist' is used to highlight the way in which Isaac and subsequent researchers have relied upon largely synchronic behavioural reconstructions to make the link between archaeological evidence and evolutionary models. The first section of this chapter explores the problems encountered in making such reconstructions.

A good example with which to identify the pitfalls of behavioural reconstruction is the archaeological research conducted to test Isaac's so-called home base hypothesis (e.g. Bunn et al. 1980, Binford 1981, Bunn and Kroll 1986, Shipman 1986, Potts 1988). Isaac $(1976 b, 1978)$ postulated that the early hominid behavioural complex included foodsharing and a division of labour. Animal foods, obtained by males, and plant foods, obtained by females, were redistributed at a central place (home base), which was also the site of stone tool manufacture. These strategies developed in evolutionary feedback with pair-bonding, increasing sociality, increasingly prolonged infant dependency and ultimately increased brain size. Isaac argued that the East African archaeological record provided evidence supporting this hypothesis. Specifically, faunal remains indicate that hominids transported meat-bearing bones away from kill sites, while their spatial association with clusters of stone tools indicates use of a central place.

The process of testing a behavioural model such as the home base hypothesis involves making a series of inferences from archaeological data. As both Potts (1988) and GiffordGonzalez (1991) make clear, these inferences are necessarily hierarchical. Following Potts' scheme, archaeologists must begin by demonstrating that sites really do exist and that they are at least partly the result of hominid activity; these are first-order or taphonomic inferences. Second-order inferences attempt to establish the specific activities of hominids: for example, did they hunt or scavenge to obtain the bones transported to sites, how much meat was available on those bones and so on. Third-order inferences make the leap from specific activities to the organization of those activities; they answer questions such as: who was hunting, were they sharing with other members of the group, was this part of a reciprocal relationship and what were the other features of that relationship? Later in this chapter it is argued that any behavioural reconstruction intended to inform debate about hominid evolution must include information about both specific activities and their wider organization. Thus useful behavioural reconstruction is only possible if sound inferences can be obtained at all three levels in Potts' hierarchy. Unfortunately, inadequacies in the archaeological data-set render third-order inference unreliable. In order to understand the nature and implications of this limit to knowledge it is necessary to investigate what we can learn from first and second-order inference.

\section{First-order inference}

Three major questions fall within the domain of first-order or taphonomic inference. First, are the clusters of bone and stone really anomalous accumulations whose existence requires explanation? Both the density of material and the faunal assemblage composition at the sites lead the majority of researchers to agree that this is indeed the case (e.g. Isaac 1984, Potts 1988, but see also Binford 1981). The second taphonomic question that must be answered concerns the duration over which the assemblages accumulated. The most 
convincing argument (Potts 1986, 1988), based on bone weathering data and the agents responsible for accumulation, is that bones were introduced to each site intermittently over a period of at least five to ten years. The third taphonomic question concerns the agent or agents of accumulation. There are three aspects: distinguishing between physical and behavioural agents, identifying which behavioural agents have at some stage been involved with the assemblages and, finally, assessing their relative contributions. The first and second tasks are relatively straightforward. Physical processes, such as water flow, appear to have played a relatively small role in assemblage formation, but both carnivores and hominids played a more substantial role (Isaac 1984, Potts 1988). The third task is altogether more difficult. Potts (1988) concedes that we will only be able to distinguish between 'primarily hominid' sites and 'primarily carnivore' sites. To place sites in the appropriate category Potts uses what he calls the contextual approach, a method that brings together the faunal and the artefactual evidence:

At the five artefact sites, tools and stone raw materials are concentrated at the same delimited locus as the bones, and these accumulations are well beyond that expected on a landscape over time through the casual discard of artefacts. In other words, these sites were specific foci to which hominids brought artefacts. The final point is that it remains doubtful at best that hominids repeatedly transported stone tools and raw materials to places of bone concentration that, if they were not primarily hominid areas of bone transport, served as major foci of bone accumulation of carnivores. Hominids, thus, are considered to have been primarily responsible for the accumulation of bones at the artefact sites.

Strictly speaking, given a five-to-ten year accumulation period, the contemporaneity of stone transport episodes and bone transport episodes cannot be demonstrated. Thus it is logically possible that hominids could have transported stone artefacts to particular locations in years when carnivores where not using them as, for example, dens. It could then be argued that the co-occurrence of stone artefacts and faunal remains is not evidence that hominids were largely responsible for bone transport at artefact sites. Even the presence of cutmarks on bones does not strictly permit refutation of this alternative scenario. Nevertheless, the interpretation that hominids and carnivores alternated over the short term in their use of the same location does seem unlikely.

Thus first-order inference allows archaeologists to state with reasonable certainty that there are anomalous clusters of faunal and artefactual material, that these accumulated over a period of five to ten years, that many of them are the result of both hominid and carnivore activity, and that hominids are largely, even if not wholly, responsible for those accumulations where stone artefacts are present. It is, however, possible to learn more.

\section{Second-order inference}

Whether hominids hunted or scavenged is a good example of the kind of question that falls within the domain of second-order inference. It is also a question which, despite considerable attention, remains unanswered. Archaeologists have available two sources of evidence about the means by which hominids acquired animal tissue: bone 
modification and body part representation. Neither provide an unambiguous distinction between hunting and primary scavenging (early access to a carcass resulting from natural death or carnivore predation). This difficulty led Potts to abandon the question of hunting or scavenging per se and to concentrate instead on the relative timing of carnivore and hominid access to animal tissue. He attempted to establish the timing of hominid access to carcasses by comparing the body part representation at primarily hominid sites with that at primarily carnivore sites (Potts 1988). This residual approach requires comparison between sites which are neither contemporary nor spatially coincident. Nevertheless, if it can be demonstrated that carnivore behaviour has remained relatively consistent across time and space then the lack of contemporaneity and spatial coincidence will not undermine the project to identify distinctly hominid carcass access patterns. Even if there are reasons to doubt the persistence of carnivore behaviour, inferences based on Potts' residual approach can be strengthened by appeal to the ecological principles for carnivore behaviour such as those that have been presented by Blumenschine (1986a, b). Thus it is possible to learn something about the ability of hominids to gain early access to carcasses. In general, it seems likely that second-order inferences which rely on comparison between hominid behaviour and other animal behaviour will be reasonably secure provided that the animal behaviour has either remained constant or can be modelled by a relatively simple set of ecological principles.

\section{Third-order inference}

While first-order inference provides reasonably secure knowledge and second-order inferences must be judged on an individual basis, all third-order inference currently appears to be unreliable. Unlike first-order inference, which is largely site based, thirdorder inference about the organization of hominid behaviour invariably requires landscape-wide knowledge of hominid activity (Isaac 1981, Potts 1988, 1989a, b, Stern 1991). Thus it must make use of data from both the high-density sites and the isolated occurrences (known as scatters) of archaeological material spread over much of the modern landscape. Several researchers, notably Blumenschine and Potts (Gibbons 1990), have initiated research with the aim of surveying a palaeolandscape in order to identify the particular topographic and ecological contexts of specific behaviours. This in turn should facilitate reconstruction of more inclusive behaviours such as food-sharing.

Such optimism is misplaced for two reasons. First, reliable inference is once again thwarted by the inadequate temporal resolution of the archaeological record. Nicola Stern's research at Koobi Fora $(1991,1993)$ has shown that an increased spatial sample of hominid activity is won at the expense of temporal control. There are some laterally extensive deposits such as the KBS and Morotot tuffs, but, presumably because they were laid down relatively quickly, they contain little archaeological material. These tuffs do provide broad chronological markers for the faunal remains and artefacts found in the sediments sandwiched between them. Broad, however, means of an order of magnitude of 100,000 years. Sedimentary units deposited between these chronological markers do not outcrop over very large areas. Consequently, a 'palaeolandscape' is typically made up of a series of discontinuous sediments such that stratigraphic correlation rapidly becomes impossible as one travels away from a given point. Thus sampling a greater range of 
topographic and ecological contexts renders the contemporaneity of their associated activities more doubtful. A reconstruction of hominid subsistence strategy based on the full range of inferred activities might well conflate some that were never practised by any one group of hominids. In this case the reconstruction would represent a hominid way of life which never actually existed.

The second potential problem with the landscape approach stems from uncertainty as to whether the archaeological record does in fact provide evidence for spatially discrete activities. It will be recalled that the first step in testing the home-base hypothesis was to establish whether the sites represent anomalous clusters of archaeological material. This chapter accepted that the methods employed led to reasonably certain knowledge that this is indeed the case. Nevertheless, it is important to understand just what was tested. The composition of the site faunal assemblages was compared with a theoretical model (derived from modern attritional death assemblages) of an untransported assemblage (Potts 1988). This comparison allowed the inference that the sites are the result of transport behaviour. In addition, the greater density of the site faunal assemblages when compared with the Plio-Pleistocene scatters allowed the further inference that transport behaviour occurred repeatedly at certain locations which we know as the sites. But, because the composition of the site faunal assemblages was not compared with that of the scatters, it cannot be inferred that, in the past, the transport behaviour which occurred repeatedly at certain locations occurred only at those locations. Similarly, site artefact assemblages were compared with the scatters in terms of density, but not composition, hence they too do not allow inference that some behaviours occurred only at certain locations in the landscape. According to Stern (1993) the scatters and sites share the same assemblage composition (but see Bunn and Kroll 1993). Consequently, the scatters and sites differ, not in the range of activities they represent, but in the frequency with which a whole suite of activities were carried out in different parts of the landscape.

\section{DEVELOPING EXPLANATIONS FOR HOMINID EVOLUTION}

Archaeologists have sought to stimulate and test explanations for hominid evolution through the intermediate goal of behavioural reconstruction. The apparent unreliability of third-order inference undermines these ultimate aims because explanations based on behavioural reconstruction require information about the wider organization of activities as well as the existence of specific activities. It is argued below that a greater understanding of the rate of hominid behavioural change is required in order to assess the reliability of third-order inference. In addition, consideration of the mechanisms for change reveals that the role of behavioural reconstruction in evolutionary explanation is not as straightforward as has generally been assumed because evolution operates at more than one level. Thus the central message is that archaeologists can no longer afford to view hominid land-use and subsistence strategies in isolation from issues of learning and cultural transmission. 


\section{Third-order inference and evolutionary explanation}

Neo-Darwinian evolutionary theory currently provides the only scientific framework within which to understand hominid evolution (Foley 1987). The central tenet of neoDarwinian theory is evolution by natural selection. Organisms better suited to their ecological niche enjoy greater reproductive success, thereby increasing the prevalence of their genotype. Providing the phenotypic properties conferring reproductive success are genetically encoded they will gradually spread through the population as a whole. Accordingly understanding of a particular evolutionary trajectory results from showing that the novel phenotypic properties increased the fitness of the organisms that possessed them, in other words that the traits were adaptive. As Ernst Mayr (1982) argues, satisfactory explanations of biological phenomena do not invoke general laws - unlike in the physical sciences - but instead employ central concepts (such as adaptation) which are articulated with the factual aspects of a given case. Hence the apparent circularity of evolutionary explanation must be overcome by a rich networking of data and concepts. In palaeoanthropology this strategy can be mobilized by predicting hominid morphology, physiology or behaviour on the basis of the selective pressures which the hominids faced. Demonstration that the predicted properties did indeed evolve confirms that the selective pressures have been correctly identified. In reality prediction and testing are conducted in a process of feedback because a phenotype is both a response to and a cause of selective pressures. This feedback process can be considered to have arrived at an adequate explanation when it meets three criteria: (1) it specifies a set of novel phenotypic features which can reasonably be regarded to confer reproductive success on an organism confronted by a specified set of selective conditions, (2) those phenotypic features actually evolved and (3) the selective conditions also existed. When the phenotypic features in question are behavioural, archaeology has a vital role in testing whether the second and third criteria are met.

Thus Gifford-Gonzalez (1991:243) states that archaeologists are engaged in 'reconstructing past states of systems [which] relate to historical narratives, both as cases demanding an evolutionary account and as challenges to existing narratives'. In other words, the goal of behavioural reconstruction is to identify the novel phenotypic properties which must be explained and to test the subsequent explanation by reconstructing the selective conditions which supposedly favoured them. At the moment this goal cannot be achieved because of the inferential problems encountered in attempting to make behavioural reconstructions. Archaeology does allow certain behaviours (phenotypic properties) to be identified, but these tend to be activities which must be placed in a wider context before they can form part of an evolutionary explanation. For example, it is reasonably certain that some hominids made stone tools and transported bones, nevertheless, these are not activities which directly increase the fitness of the individuals engaged in them. Evolutionary explanations require an assessment of adaptive value, but this is not an intrinsic property of the kinds of behaviour identified through first-order inference. Adaptive value is a property of the more inclusive behaviours which are reconstructed through second- and third-order inference. Some second-order inferences can be made without a debilitating degree of uncertainty (the example discussed above was the timing of access to animal tissue). 
Even so, this extra information is insufficient to test an evolutionary explanation. Imagine that the use of stone tools enabled hominids to detach meat-bearing bones and transport them away from the kill site for consumption in relative safety. Intuitively, this scenario confers an adaptive advantage on its protagonists because it increases the efficiency of their foraging behaviour which in turn can be expected to contribute to increased reproductive success (Dunbar 1988). Whether this intuition is correct, however, depends upon the wider behavioural context of the foraging strategy in question: is it part of a group effort to drive away the initial carnivore consumers, does it involve sharing the spoils, does it increase reproductive success through provisioning the young or through increased sexual access, and so on. A satisfactory evolutionary explanation is one which shows that a behaviour was adaptive by describing how it was adaptive. Unfortunately the requisite wider behavioural context can only be reconstructed through third-order inference which, as has been argued, is currently unreliable.

It will be recalled that the major problem undermining third-order inference is the need to conflate behavioural episodes across tens or possibly hundreds of thousands of years. This renders inference about wider behavioural organization unreliable owing to the risk of conflating activities from separate land-use or subsistence strategies into one strategy which never actually existed. The notion of risk serves to emphasize that the unreliability of third-order inference stems as much from archaeologists' ignorance of the quality of data required to support their interpretations as it does from the actual quality of the data which is available. For instance, it could be that hominid behaviour changed so slowly that conflating activities separated by tens of thousands of years would not result in an erroneous reconstruction of hominid behavioural adaptation. Equally, the converse might be true. Clearly then, given the nature of the archaeological record, the reliability of thirdorder inference depends upon the rate of hominid behavioural change. In order to understand how rapidly hominid behaviour might have changed it is necessary to reconsider the nature of the evolutionary process.

\section{Mechanisms for behavioural change}

Biologists and philosophers of science writing from an evolutionary epistemological perspective (e.g. Campbell 1974, Hull 1982, Odling-Smee 1983) have argued that evolution by natural selection is just one implementation of a more general process of knowledge gain which occurs at several levels. At each level a common algorithm is implemented through a unique mechanism. The common algorithm is a continuous alternation of processes which generate variety and processes which winnow that variety by selection and retention. Odling-Smee (1983) describes four levels of knowledge gain each characterized by a specific mechanism: at level 1 populations gain knowledge through the genetic mechanism of evolution by natural selection; at levels 2 and 3 organisms gain knowledge through variable epigenesis and individual learning respectively; and finally, at level 4 , it is once again populations which gain knowledge, but this time through the mechanism of cultural evolution. All four mechanisms result in their corresponding unit of knowledge-gain acquiring a better way of existing in a given environment. Nevertheless, one important way in which they differ is in the rate and scope of the resulting change. For instance, the major morphological and physiological 
changes required for hominid bipedalism evolved relatively slowly through natural selection. Individual hominids probably benefited from a more rapid, but equally irreversible, fine-tuning of those changes through variable epigenesis. By contrast, individual learning would have provided hominids with the ability to alter their behaviours reflexively over a relatively short timescale. Finally, any persistence of reflexively learned behaviour across time and space may have been the result of cultural learning.

This evolutionary epistemological perspective suggests that the maximum rate at which hominid land-use and subsistence strategies changed would have depended upon whether they were genetically determined, individually learned or culturally learned. Given that the viability of pursuing evolutionary explanation through behavioural reconstruction depends upon the reliability of third-order inference, and that this in turn depends upon the rate of change of hominid behaviour, it is imperative that archaeologists investigate the balance between individual and cultural learning present at different stages in hominid evolution. The next section suggests how this might be achieved through the use of computer simulation. But first, identification of the mechanisms underlying hominid behavioural change carries another implication for the role of behavioural reconstruction in evolutionary explanation.

The balance between individual and cultural learning at different stages in hominid evolution alters the requirements for an adequate evolutionary explanation. For instance, suppose that early hominids did not possess a genetic blueprint for a neurologically hardwired programme which instructed them in the art of using stone tools to gain early access to meat. Instead they evolved the neurological capacity for complex and flexible behaviour (Foley 1987), that is, an increased degree of individual and possibly cultural learning. This capacity for complex and flexible behaviour evolved through natural selection, but thereafter the existence of many specific behaviours would only be immediately explicable in terms of faster mechanisms of information gain. According to this scenario, hominid evolution became a two-tier process whose explanation involves reconciling the immediate causality of organism learning with the ultimate causality of natural selection. Thus it is no longer adequate to show that and how particular hominid behaviours were adaptive; in addition it must be demonstrated that and how the particular way in which these behaviours 'evolved' was adaptive. Explanations for each of the two tiers of hominid evolution must be tested over different timescales. In the case of the evolution of flexible behaviour the adaptation that results from genetic evolution is not a particular behaviour or suite of behaviours, but a means of controlling and altering behaviour. Thus demonstrating the adaptive value of individual learning requires showing that and how learned behaviour varies with changing selective conditions. By contrast, the adaptation that results from organism learning is a particular behaviour or suite of behaviours whose adaptive value is demonstrated in the context of the particular selective conditions which favoured them. Consequently, it can be seen that the use of behavioural reconstruction to test evolutionary explanations requires at least two series of reconstructions of hominid behaviour, each generated over a different time-frame. 


\section{THE SIMULATION OF INDIVIDUAL LEARNING AND CULTURAL LEARNING IN HOMINID EVOLUTION}

Information about the mechanisms and rate of hominid behavioural change is required both to assess the reliability of third-order inference and to ensure that behavioural reconstructions are made at the appropriate timescales. The remainder of this chapter describes the use of computer simulation to explore the role of individual and cultural learning in hominid evolution. Both Mithen (1991) and Stern (1991) have noted the potential utility of simulation for hominid studies, but despite their observations the only published example remains Potts' (1988) investigation of the energetic costs of different bone transport strategies. Nevertheless, computer simulation provides the ideal method for exploring the cumulative effect of differently scaled processes because its experimental nature allows the investigator to alter the rates of those processes and the way in which they interact.

The term 'individual learning' refers to the process by which individual hominids learn about their natural and social environment through their own trial and error. In other words, based on their own knowledge, they make decisions about the appropriate course of action, for example where to forage, and then adjust that knowledge in the light of subsequent experience. Cultural transmission, on the other hand, refers to the outcome of cultural learning, a process in which hominids acquire information from and/or copy the decisions of others. The extent to which individual learning and cultural learning are distinct at a psychological level is currently the subject of considerable debate (e.g. Tomasello et al. 1993). Nevertheless, at a phenomenological level there can be little doubt that the two are distinct, both in terms of their effect and in terms of the associated costs to the learner.

The effect and significance of cultural learning has been succinctly described by Tomasello et al. (1993:495):

Many animal species live in complex social groups; only humans live in cultures. Cultures are most clearly distinguished from other forms of social organisation by the nature of their products - for example, material artefacts, social institutions, behavioural traditions, and languages. These cultural products share, among other things, the characteristic that they accumulate modifications over time.

By contrast, behaviours and material artefacts resulting from individual learning must be constantly reinvented if they are to persist across space and time, and there is no sense in which they can accumulate modifications. Thus one might expect a subsistence strategy based on individual learning to show greater variability across space and time and little or no cumulative change. Persistence of individually learned traits will only be found where there are strong external constraints such as genetic determination, the properties of available raw materials, or very specific problems posed by the environment.

Individual learning and cultural learning also differ in their costs to the learner. This is true even if, as Heyes and Plotkin argue (1989), the process of cultural learning is 
psychologically little different from that of other learning forms. As they point out, "what one [cultural learner] learns is in part a function of what some other person or animal has learned' (1989:149); consequently, while possibly subject to the same cognitive costs as the individual learner, the cultural learner will not incur the energetic costs, loss of time and risk associated with trial-and-error learning. Admittedly this is only true if the learned information or behaviour is both correct and relevant-a point to which I will return - but assuming that this condition is fulfilled it would, for example, be possible for a hominid to forage in rich resource patches without wasting time and energy sampling poor patches. This cost differential provides the basis for understanding the conditions under which a propensity for cultural learning might be expected to evolve by natural selection.

Boyd and Richerson (1985) have analysed the conditions favouring the evolution of cultural learning. As just noted, there is little to be gained from acquiring information which is inaccurate or out of date. Boyd and Richerson's analysis suggests that a propensity for cultural learning should evolve where the spatial and temporal heterogeneity of an environment is neither so great that culturally learned information is misleading, nor so little that the genetic mechanism provides a cheaper (since it does not incur the developmental and metabolic costs of a large brain) way of storing and altering information. Thus, in order to establish likely rates of behavioural change, archaeologists need to ascertain what balance of individual and cultural learning would have been favoured in the environments inhabited by the hominids in question. Boyd and Richerson's analysis provides a strong theoretical basis for this investigation; however, from an archaeological perspective it presents two practical problems. The first is that Boyd and Richerson characterize environmental heterogeneity in terms of an autocorrelation coefficient: unfortunately it is difficult to estimate this parameter from palaeoenvironmental reconstruction. The second problem concerns individual learning. It has already been noted that the viability of cultural learning depends upon the quality of the information which is learned, and that one factor affecting this is the degree of environmental heterogeneity. Another factor is the accuracy of the individual learning that originally generated the information later culturally transmitted. Boyd and Richerson characterize the error in individual learning as the variance of a normally distributed random variable: needless to say, the variance of error terms in hominid knowledge is simply unknown.

The challenge facing archaeologists is to build a computer simulation model which embodies the principles of Boyd and Richerson's formal model, but allows investigation of the effect of individual learning abilities on foraging in environments characterized by the type of information which is actually available from palaeoenvironmental reconstruction. Figure 6.1 is a flow-chart depicting just such a simulation. In the inner shell hominids forage in an environment made up of resource patches, each of which is characterized by a reward with an associated probability and a cost of search. This latter allows the model to incorporate factors such as temperature stress and the need for vigilance against predators. In the course of foraging hominids must decide which patches to exploit on the basis of their knowledge about those patches. Hominid decision criteria are modelled using principles derived from optimal foraging theory (see, for example Stephens and Krebs 1986) although it is not assumed that they make optimal 
decisions, instead they satisfice, that is, make 'good enough' decisions (Mithen 1990:45). The simulation model assumes that hominids have reasonable knowledge of the value of resources that they might encounter; what they have to learn is the probability of encounter. The way in which a given hominid learns about

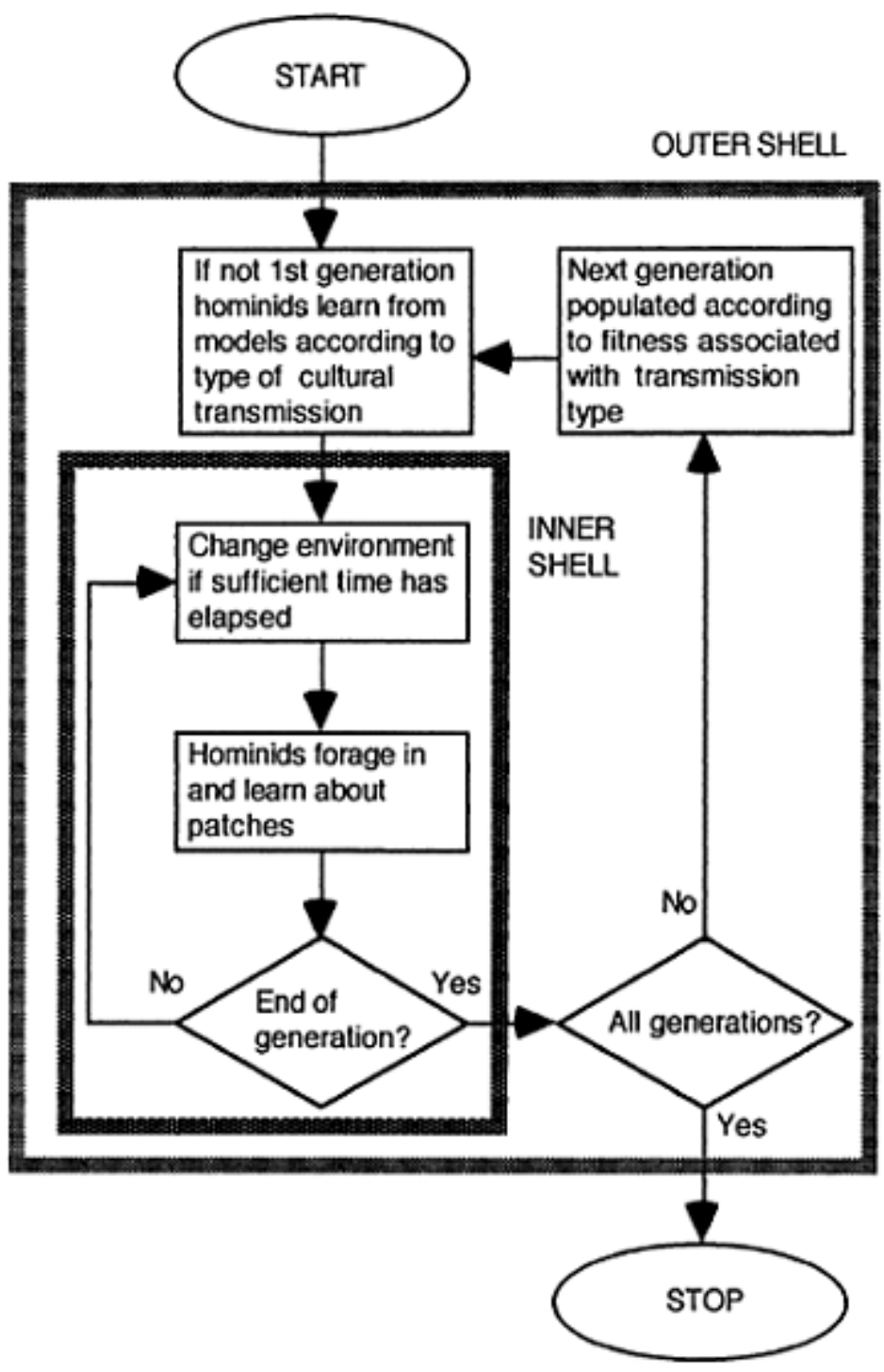

Figure 6.1 Flow-chart of a computer simulation model incorporating Boyd and Richerson's formal principles, but permitting investigation of the effect of individual learning abilities in realistic foraging environments 
its environment depends upon whether its 'genotype' specifies a strong propensity for individual learning or a strong propensity for cultural learning. In the case of individual learning the hominid adjusts its expected probability of encounter for each resource according to how often it has encountered the resource on previous visits to the patch. In the case of cultural learning the hominid imitates the foraging behaviour of another hominid who appears to be fitter than average; this is an implementation of what Boyd and Richerson term 'indirect biased transmission' (1985:135). The outer shell of the model allows the relative frequency of the two 'genotypes' for learning propensity to vary by natural selection, that is, according to their relative fitness in the population.

It must be stressed that experimentation with the simulation model is not yet complete, thus the following discussion is intended as an illustration of potential rather than a rigorous presentation of firm conclusions. Figure 6.2 shows the change over 200 generations in relative frequency of hominids possessing the 'genotype' for cultural learning (indirect biased transmission). The population was initialized with 50 per cent of 'genotypes' specifying cultural learning and 50 per cent specifying individual learning. It was then allowed to evolve in environments ranging from temporally homogeneous to temporally heterogeneous (characterized by high and low autocorrelation coefficients respectively). It can be seen that temporal homogeneity favours cultural learning while temporal heterogeneity favours individual learning. This makes sense because in a temporally homogeneous environment information and behaviours which are beneficial to one generation are likely to be beneficial to the next, thus learning from the previous generation is a viable shortcut enabling its practitioners to divert more time and energy to provisioning the young or finding a mate. By contrast, in a temporally heterogeneous environment hominids who adhere to the knowledge and behaviours of the previous generation are likely to have committed themselves to a life of costly error. These runs of the computer simulation were made using environments of known autocorrelation in order to establish the theoretical validity of the simulation model by checking that evolution occurs in the manner predicted by Boyd and Richerson's formal model. Once the simulation model has been validated in this way it can be put to use by inputting a series of more realistic palaeoenvironmental parameters in order to establish what balance of the two learning types would have been favoured in hominid populations.

The second reason cited above for building a simulation model of hominid individual and cultural learning was that it would facilitate investigation of the relationship between individual learning ability and the viability of cultural learning. Figure 6.3 suggests that the quality of individual learning does alter the viability of cultural learning. The quality of individual learning has been modelled in terms of the amount of information a hominid uses in learning what to expect from a given patch. While it can be argued that this does not constitute a model of different cognitive abilities in the sense of different computational abilities, it does have the virtue of modelling differing rates of tracking the environment, and it is this which is most likely to affect the viability of cultural transmission since, as the evolutionary epistemological perspective reveals, different learning mechanisms are geared to coping with different rates of change. Figure 6.3 


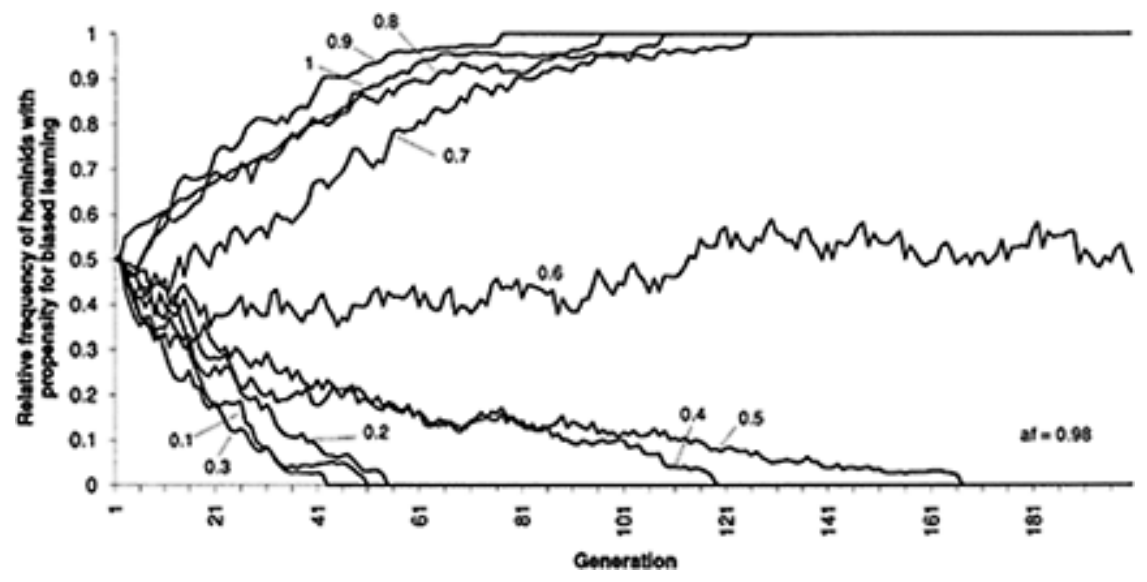

Figure 6.2 Change in relative frequency of hominids with biased transmission according to autocorrelation of environmental change

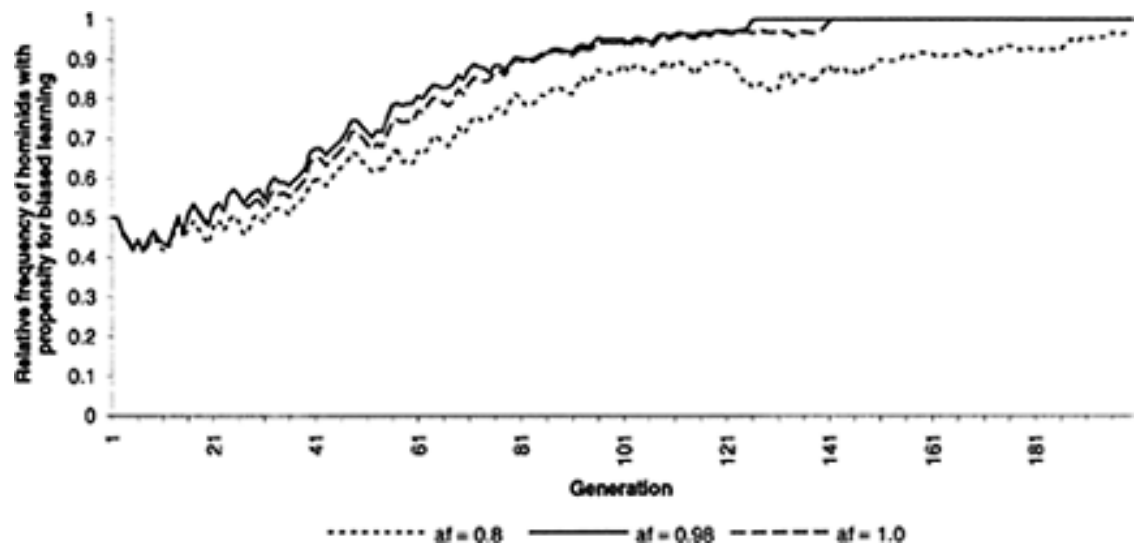

Figure 6.3 Selective pressure for cultural learning is affected by attention paid to past experience

shows that the rate at which the 'genotype' for cultural learning spreads through a population depends upon the extent to which hominids make use of past experience when individually learning about their environment. Cultural learning is most strongly favoured in the population whose members pay about twice as much attention to their most recent experience compared to their experience of 30 days ago $(\mathrm{af}=0.98)$. Cultural learning is less strongly favoured in populations where hominids pay either more $(a f=1.0)$ or less $(\mathrm{a} f=0.8)$ attention to past experience.

Figure 6.4 provides some clues as to why cultural learning is less favoured in populations of hominids paying either very considerable or very little attention to past experience. The graph shows how closely a hominid is able to track a changing 
environment according to the amount of attention it pays to past experience. Clearly there is a trade-off between the speed of response to environmental change and the precision of the knowledge which a hominid gains. In the following discussion a distinction is made between accuracy and precision. The former refers to the extent to which hominid estimates cluster around the true value of the probability of encounter as opposed to some other (erroneous) value. The latter refers to the degree of dispersion around the mean value of the estimates, irrespective of whether that value is close to the true value. Figure 6.6

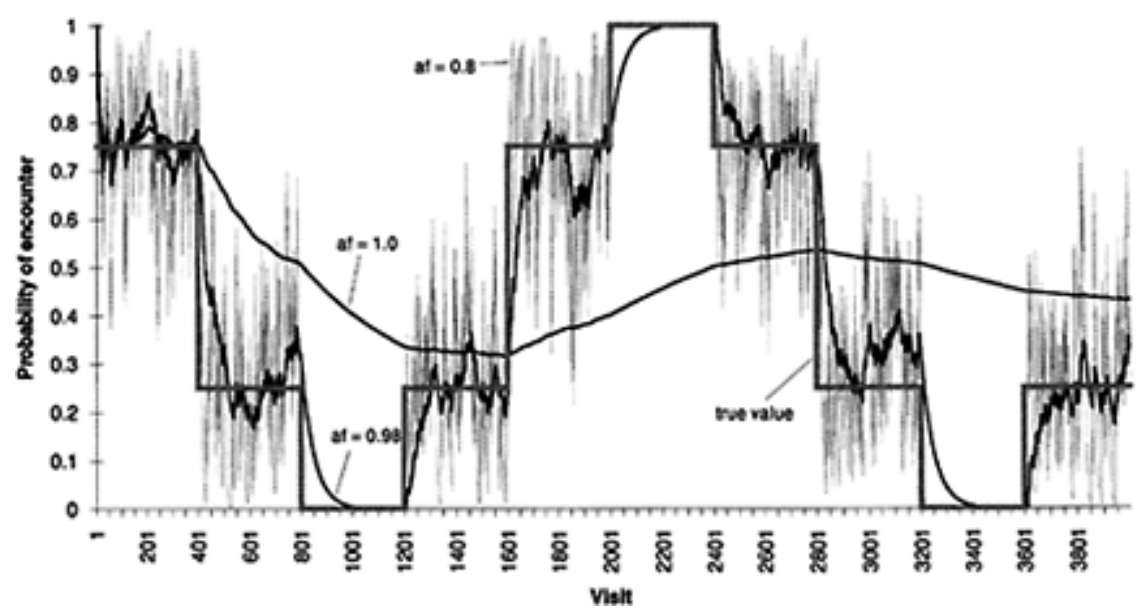

Figure 6.4 Effect of memory on hominid's ability to model environment

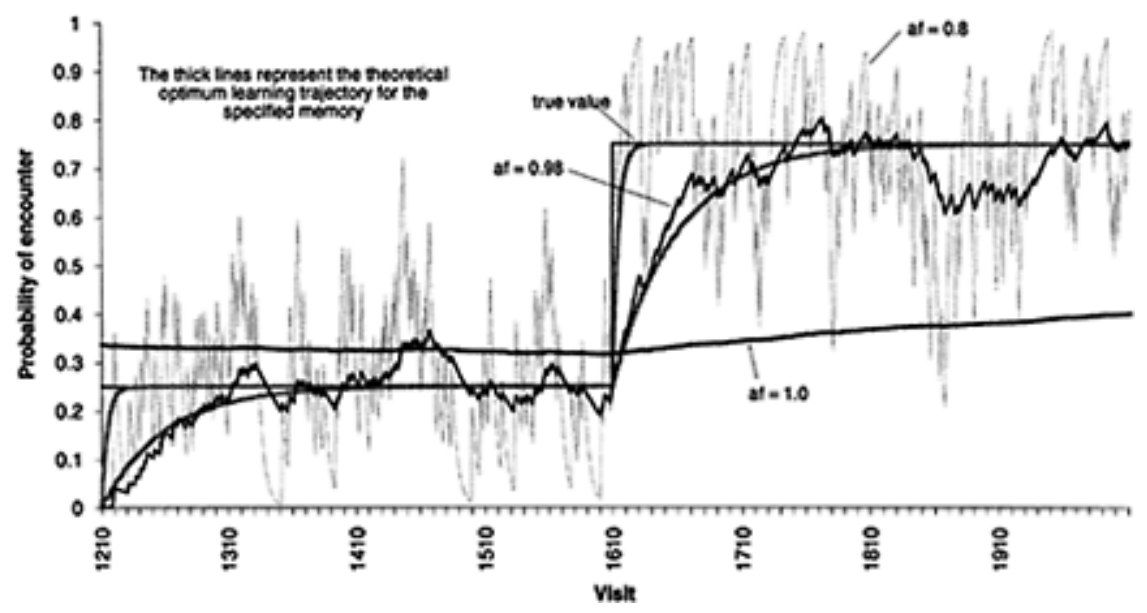

Figure 6.5 Hominid tracking changing environment

illustrates how accuracy and precision combine to influence the overall quality of the hominid's knowledge (better knowledge is represented by a lower standard deviation). 
The three sets of statistics relate to the entire duration of Figure 6.4, but are better understood if viewed alongside Figure 6.5. The thin lines in Figure 6.5 represent a single hominid's actual estimates of the new probability of encounter. The thick lines represent the optimum adjustment towards the changed probability of encounter based solely on the elapsed time since the environmental change. This optimum provides a close approximation to a moving average of the actual hominid estimates; thus the distance between the optimum and the true value provides a convenient measure of the accuracy of the hominids knowledge. Likewise, the distance between the optimum and actual estimates provides a measure of the precision of the hominids knowledge. These distances are given as standard deviations in Figure 6.6, which clearly shows that while paying much attention to past experience $(\mathrm{af}=1.0)$ results in a precise estimate of the environment, that estimate may actually be highly inaccurate if the environment is changing. On the other hand, paying much less attention to past experience $(\mathrm{af}=0.8)$ improves the accuracy of the estimate, but drastically reduces its precision. The best compromise turns out to be the degree of attention to past experience which most strongly favoured cultural learning ( $\mathrm{af}=0.98$ ).

By using the computer simulation just described it should be possible to draw some conclusions regarding the balance between individual and cultural learning at different stages in hominid evolution. This will be

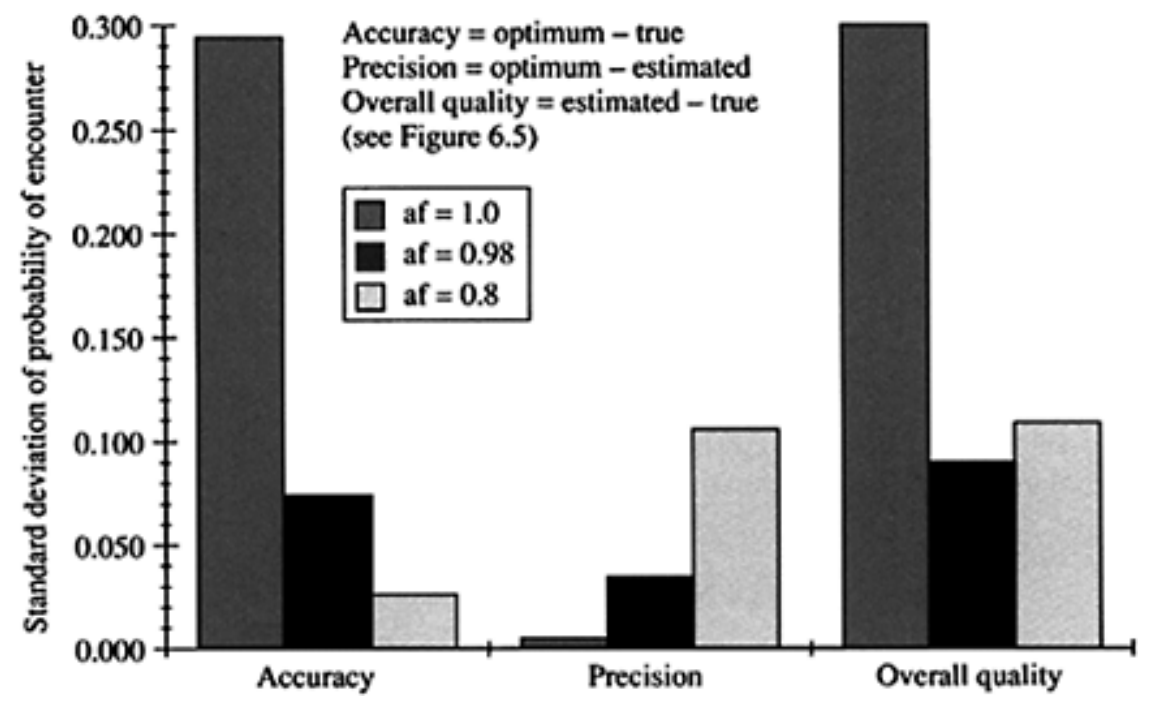

Figure 6.6 Standard deviation as a measure of the accuracy and precision of hominid model of environment

achieved by running the simulation with environmental parameters similar to those of the palaeoenvironments in which hominids were living. It should be noted that specification of such parameters does not require knowledge of exact probabilities of encounter of different resource types in the palaeoenvironment, but rather a more general understanding of magnitudes and rates of change of such probabilities. In addition, as is 
already evident from the results described above, the balance between individual and cultural learning favoured in the simulations will partly depend on the wider cognitive abilities attributed to the hominids. These abilities must be compatible with information gleaned from the study of hominid stone tool technology and ape cognition (see, for example, papers in Gibson and Ingold 1993). The balance between individual and cultural learning established in the simulations can then be used to assess the reliability of third-order inference, and thus the viability of using behavioural reconstruction to stimulate and test evolutionary explanations.

\section{CONCLUSION}

Archaeologists will not be able to fulfil their aim of contributing to our understanding of hominid evolution in the absence of a greater understanding of the mechanisms and rate of hominid behavioural change. This is not to suggest that all aspects of the current research paradigm are inappropriate. First- and second-order inferences remain important because they provide reasonably certain knowledge about some specific hominid activities such as tool-making and bone transport. Nevertheless, it is essential that the utility of third-order inference be reassessed through two lines of research. The first is an investigation of the timescales over which behavioural reconstructions are required in order to test evolutionary models. The second must establish whether the structure of the archaeological record permits reliable inference over the requisite timescales. While the latter involves the sort of detailed field study reported at length by Nicola Stern (1991, 1993), research into the rate of hominid behavioural change is still in its infancy. This chapter has argued that the appropriate timescales for behavioural reconstruction can only be determined through an exploration of the balance between individual and cultural learning in hominid evolution. Computer simulation will play a valuable role in this endeavour.

\section{ACKNOWLEDGEMENTS}

I am grateful to Nikki Stern for the invitation to join her at Koobi Fora, for numerous stimulating discussions about hominid archaeology, and for comments on an earlier draft of this chapter. I was able to gain first-hand experience of the nature of the East African Lower Pleistocene archaeological record with the help of financial support from the Science and Engineering Research Council, the Boise Fund (University of Oxford), King's College Cambridge and the Dorothy Garrod Travel Fund (University of Cambridge). I also thank Stephen Shennan and James Steele for inviting me to contribute to this book and for their advice. Finally, I am particularly grateful to Steven Mithen for his encouragement and for asking some simple but awkward questions. 


\section{REFERENCES}

Binford, L. (1981) Bones: Ancient Men and Modern Myths. New York: Academic Press. Blumenschine, R.J. (1986a) Early Hominid Scavenging Opportunities. British Archaeological Reports International Series 283. Oxford: B.A.R.

Blumenschine, R.J. (1986b) 'Carcass consumption sequences and the archaeological distinction of scavenging and hunting.' Journal of Human Evolution 15: 639-660.

Boyd, R. and Richerson, P.J. (1985) Culture and the Evolutionary Process. Chicago: Chicago University Press.

Bunn, H.T. and Kroll, E. (1986) 'Systematic butchery by Plio-Pleistocene hominids at Olduvai Gorge, Tanzania.' Current Anthropology 27:431-452.

Bunn, H.T. and Kroll, E. (1993) 'CA comment on N.Stern. The Structure of the Lower Pleistocene Archaeological Record: a case study from the Koobi Fora formation.' Current Anthropology 34:216-217.

Bunn, H.T., Harris, J.W.K., Isaac, G.L., Kaufulu, Z., Kroll, E., Schick, K., Toth, N. and Behrensmeyer, A.K. (1980) 'FxJj50: an early Pleistocene site in northern Kenya.' World Archaeology 12:109-136.

Campbell, D.T. (1974) 'Evolutionary epistemology.' In P.A.Schlipp (ed.) The Philosophy of Karl Popper Book 1. La Salle, IL: The Open Court Publishing Company.

Dunbar, R.I.M. (1988) Primate Social Systems. Beckenham, England: Croom Helm.

Foley, R.A. (1987) Another Unique Species: Patterns in Human Evolutionary Ecology. Harlow: Longman Scientific and Technical.

Gibbons, A. (1990) 'Palaeontology by bulldozer (research news).' Science 247: 14071409.

Gibson, K.R. and Ingold, T. (eds) (1993) Tools, Language and Cognition in Human Evolution. Cambridge: Cambridge University Press.

Gifford-Gonzalez, D. (1991) 'Bones are not enough: knowledge and interpretative strategies in zooarchaeology.' Journal of Anthropological Archaeology 10: 215-254.

Heyes, C.M. and Plotkin, H.C. (1989) 'Replicators and interactors in cultural evolution.' In M.Ruse (ed.) What the Philosophy of Biology Is, pp. 139-162. Dordrecht: Kluwer Academic Publishers.

Hull, D.L. (1982) 'The naked meme.' In H.C.Plotkin (ed.) Development and Culture: Essays in Evolutionary Epistemology, pp. 273-327. Chichester: Wiley.

Isaac, G. (1969) 'Studies of early culture in East Africa.' World Archaeology 1: 1-28.

Isaac, G. (1972) 'Early phases of human behaviour: models in lower Palaeolithic archaeology.' In D.L.Clarke (ed.) Models in Archaeology, pp. 167-199. London: Methuen.

Isaac, G. (1976a) 'Early hominids in action: a commentary on the contribution of archaeology to understanding the fossil record of east Africa.' Yearbook of Physical Anthropology for 1975, pp. 19-35. Washington DC: AAPA.

Isaac, G. (1976b) 'The activities of early African hominids.' In G.Isaac and E. McCown (eds) Human Origins, pp. 483-514. Menlo Park, CA: Benjamin.

Isaac, G. (1978) 'The food sharing behaviour of proto-human hominids.' Scientific American 238:90-108.

Isaac, G. (1981) 'Stone age visiting cards: approaches to the study of early land-use patterns.' In I.Hodder, G.Isaac and N.Hammond (eds) Pattern of the Past, pp. 131155. Cambridge: Cambridge University Press. 
Isaac, G. (1983) 'Aspects of human evolution.’ In D.S.Bendall (ed.) Evolution from Molecules to Men, pp. 509-543. Cambridge: Cambridge University Press.

Isaac, G. (1984) 'The archaeology of human origins: studies of the Lower Pleistocene in east Africa 1971-1981.' Advances in Old World Archaeology 3: 1-87.

Mayr, E. (1982) The Growth of Biological Thought: Diversity, Evolution and Inheritance. Cambridge, MA: Belknap Press.

Mithen, S.J. (1990) Thoughtful Foragers: A Study of Prehistoric Decision-making. Cambridge: Cambridge University Press.

Mithen, S.J. (1991) 'Home bases and stone caches: the archaeology of early hominid activities (review article).' Cambridge Archaeological Journal 1: 277-283.

Odling-Smee, F.J. (1983) 'Multiple levels in evolution: an approach to the nature-nurture issue via applied epistemology.' In G.C.L.Davey (ed.) Animal Models of Human Behaviour, pp. 135-158. Chichester: Wiley.

Potts, R. (1986) 'Temporal span of bone accumulations at Olduvai Gorge and implications for early hominid foraging behaviour.' Palaeobiology 12:25-31.

Potts, R. (1988) Early Hominid Activities at Olduvai. New York: Aldine de Gruyter.

Potts, R. (1989a) 'Ecological context and explanations of hominid evolution.' Ossa 14:99-112.

Potts, R. (1989b) 'Olorgesailie: new excavations and findings in Early and Middle Pleistocene contexts, southern Kenya rift valley.' Journal of Human Evolution 18:477484.

Shipman, P. (1986) 'Scavenging or hunting in early hominids: theoretical framework and tests.' American Anthropologist 88:27-43.

Stephens, D.W. and Krebs, J.R. (1986) Foraging Theory. Princeton: Princeton University Press.

Stern, N. (1991) 'The scatters between the patches: a study of early hominid land-use patterns in the Turkana Basin, Kenya.' Unpublished PhD dissertation, Harvard University.

Stern, N. (1993) 'The structure of the Lower Pleistocene archaeological record: a case study from the Koobi Fora formation.' Current Anthropology 34:201-225.

Tomasello, M., Kruger, A.C. and Ratner, H.H. (1993) 'Cultural learning.' Behavioural and Brain Sciences 16:495-552. 